\title{
$S$-Manifolds Versus Indefinite $S$-Manifolds and Local Decomposition Theorems
}

\author{
Letizia Brunetti, Anna Maria Pastore*
}

(Communicated by Aurel BEJANCU)

\begin{abstract}
To any globally framed $f$-manifold carrying a structure of $\mathcal{S}$-manifold we associate several indefinite $\mathcal{S}$-manifolds. We determine the links between the corresponding Levi-Civita connections and sectional curvatures. We state some local semi-Riemannian decomposition theorems.
\end{abstract}

Keywords: Semi-Riemannian manifolds; indefinite metrics; Lorentz metrics; $f$-structures.

AMS Subject Classification (2010): Primary: 53C50; Secondary: 53C15, 53D10.

\section{Introduction}

Globally framed $f$-manifolds, also known as $f$-manifolds with parallelizable kernel (f.pk-manifolds), represent a natural generalization of almost contact manifolds, $[12,11,20]$. Such manifolds have been studied by several authors and from different points of view, $[3,7,9,15,17]$. They are manifolds $M^{2 n+s}$ equipped with an $f$-structure $\varphi$ of rank $2 n$ with kernel parallelizable by $s$ vector fields $\xi_{1}, \ldots \xi_{s}$. Such manifolds admit Riemannian metrics $g$ which verify the so-called compatibility condition: $g(\varphi X, \varphi Y)=$ $g(X, Y)-\sum_{i=1}^{s} \eta^{i}(X) \eta^{i}(Y)$, where $\eta^{1}, \ldots, \eta^{s}$ are 1-forms dual to $\xi_{1}, \ldots \xi_{s}$. When the so-called normality tensor field $N:=[\varphi, \varphi]+2 \sum_{i=1}^{s} d \eta^{i} \otimes \xi_{i},[\varphi, \varphi]$ being the Nijenhuis torsion of $\varphi$, vanishes and the Sasaki 2-form $\Phi=g(-, \varphi-)$ is closed, one obtains a class of manifolds that generalizes quasi-Sasakian manifolds. They are called $\mathcal{K}$-manifolds by D.E. Blair in [2]. Two special subclasses are also defined: $\mathcal{S}$-manifolds, by requiring that $d \eta^{1}=\cdots=d \eta^{s}=\Phi$, and $\mathcal{C}$-manifolds, by requiring that $d \eta^{1}=\cdots=d \eta^{s}=0$.

In [4] we studied a generalization of such $\mathcal{S}$-manifolds, called indefinite $S$-manifolds.

Furthermore, indefinite Sasakian manifolds have been widely studied by many authors and in [6] we studied Lorentz Sasakian manifolds obtaining a classification result for compact Lorentz Sasakian space forms.

After a section collecting the fundamental data on globally framed $f$-manifolds, Section 3 is devoted to the construction of indefinite $\mathcal{S}$-manifolds associated to an $\mathcal{S}$-manifold. In Section 4 we discuss the effects of $D$-homothetic transformations. Then, in Section 5 we state some local decomposition theorems and we end discussing, in Section 6, the so-called special $\mathcal{S}$-manifolds.

Manifolds, tensor fields and maps are assumed to be smooth, and all manifolds are supposed to be connected. Moreover, $\mathfrak{X}(M)$ will denote the Lie algebra of vector fields on a manifold $M$. Finally, we adopt the notation in [14] for the curvature tensor field and the exterior differentiation.

\section{Preliminaries}

A $(2 n+s)$-dimensional manifold $M$ is called a globally framed $f$-manifold (briefly $g . f . f$-manifold) if it is endowed with a (1,1)-tensor field $\varphi$ of constant $\operatorname{rank} 2 n$, such that $\operatorname{ker} \varphi$ is parallelizable i.e. there exist global vector fields $\xi_{i}, 1 \leq i \leq s$, and 1-forms $\eta^{i}$, satisfying $\eta^{i}\left(\xi_{j}\right)=\delta_{j}^{i}$ and $\varphi^{2}=-I+\sum_{i=1}^{s} \eta^{i} \otimes \xi_{i}$.

A g.f.f-manifold $\left(M^{2 n+s}, \varphi, \xi_{i}, \eta^{i}\right), 1 \leq i \leq s$, is said to be an indefinite $g . f . f$-manifold if $g$ is a semi-Riemannian metric on $M^{2 n+s}$ satisfying the so-called compatibility condition $g(\varphi X, \varphi Y)=g(X, Y)-\sum_{i=1}^{s} \varepsilon_{i} \eta^{i}(X) \eta^{i}(Y)$ for any $X, Y \in \mathfrak{X}\left(M^{2 n+s}\right)$. Here $\varepsilon_{i}= \pm 1$ according to the spacelike or timelike causal character of $\xi_{i}$. Then, 
for $1 \leq i \leq s$, one has $\eta^{i}(X)=\varepsilon_{i} g\left(X, \xi_{i}\right)$, ([4]). A special class of indefinite $g . f . f$-manifolds consists on the indefinite $\mathcal{S}$-manifolds i.e. normal indefinite $g$.f.f-manifolds such that $d \eta^{i}=\Phi$, for any $i \in\{1, \ldots, s\}$, where $\Phi(X, Y)=g(X, \varphi Y)$ for any $X, Y \in \mathfrak{X}\left(M^{2 n+s}\right)$. The normality condition is expressed by the vanishing of the tensor field $N=[\varphi, \varphi]+2 \sum_{i=1}^{s} d \eta^{i} \otimes \xi_{i},[\varphi, \varphi]$ being the Nijenhuis torsion of $\varphi$. Furthermore, as proved in [4], the Levi-Civita connection of an indefinite $\mathcal{S}$-manifold satisfies:

$$
\left(\nabla_{X} \varphi\right) Y=g(\varphi X, \varphi Y) \bar{\xi}+\bar{\eta}(Y) \varphi^{2}(X)
$$

where $\bar{\xi}=\sum_{i=1}^{s} \xi_{i}$ and $\bar{\eta}=\sum_{i=1}^{s} \varepsilon_{i} \eta^{i}$.

We remark that when each $\varepsilon_{i}=1$, we are in the setting of $\mathcal{S}$-manifolds.

We recall that the $\xi_{i}$ 's are Killing vector fields, $\nabla_{X} \xi_{i}=-\varepsilon_{i} \varphi X$ and $\operatorname{ker} \varphi$ is an integrable flat distribution since $\nabla_{\xi_{i}} \xi_{j}=0$. In [4] we described two different indefinite $\mathcal{S}$-structures with a metric of index $\nu=2$ on $\mathbb{R}^{6}$ and an indefinite $\mathcal{S}$-structure with Lorentz metric on $\mathbb{R}^{4}$. Clearly, for $s=1$, we reobtain indefinite Sasakian manifolds and when the index $\nu(g)=0$, then we obtain the notion of $\mathcal{S}$-manifold. An (indefinite) $\mathcal{S}$-manifold is never flat since the mixed sectional curvatures are given by ( $X$ non lightlike) $K\left(X, \xi_{i}\right)=\varepsilon_{i}$.

In [4] we proved that the $\varphi$-sectional curvatures completely determine the sectional curvatures of an indefinite $\mathcal{S}$-manifold. Moreover, for the Riemannian curvature tensor field $R$, we gave the expression which characterizes the pointwise constancy of the $\varphi$-sectional curvature.

Finally, we recall that an indefinite $\mathcal{S}$-manifold is said to be special if $\varepsilon=\sum_{i=1}^{s} \varepsilon_{i}=0$, i.e. $s=2 p$ and, without loss of generality, we suppose that $\xi_{1}, \ldots, \xi_{p}$ are timelike vector fields, while $\xi_{p+1}, \ldots, \xi_{2 p}$ are spacelike vector fields, [4].

\section{From $\mathcal{S}$-manifolds to indefinite $\mathcal{S}$-manifolds}

It is well known, [16], that one can define a Lorentz metric on a Sasakian manifold $\left(M^{2 n+1}, \varphi, \xi, \eta, g\right)$ putting $\widetilde{g}=g-2 \eta \otimes \eta$.

Then it is easy to check that $M^{2 n+1}$ equipped with the structure $(\varphi, \xi, \eta, \widetilde{g})$ becomes an indefinite Sasakian manifold, more precisely a Lorentz Sasakian manifold. In [6] we proved the following result.

Theorem 3.1. Let $\left(M^{2 n+1}, \varphi, \xi, \eta, g\right)$ be a Sasakian manifold, $\left(M^{2 n+1}, \varphi, \xi, \eta, \tilde{g}\right)$ the associated Lorentz Sasakian manifold. Then the Sasakian manifold has constant $\varphi$-sectional curvature $c \in \mathbb{R}$ if and only if the Lorentz Sasakian manifold has constant $\varphi$-sectional curvature $\tilde{c}=c+6$ and we have

$$
c>-3 \Leftrightarrow \tilde{c}>3, \quad c=-3 \Leftrightarrow \tilde{c}=3, \quad c<-3 \Leftrightarrow \tilde{c}<3 .
$$

Then we classified compact Lorentz Sasakian manifolds of constant $\varphi$-sectional curvature, by using the results stated by Tanno [18],[19] and the following result stated by Guediri and Lafontaine in [13].

Theorem 3.2. Let $(M, g)$ be an $n$-dimensional compact semi-Riemannian manifold with signature $(n-p, p)$ where $2 p \leq n$. If there exist $p$ Killing vector fields, linearly independent everywhere and timelike, then $(M, g)$ is geodetically complete.

Now we are looking to extend the above constructions to $\mathcal{S}$-manifolds obtaining indefinite $\mathcal{S}$-manifolds.

Let $\left(M^{2 n+s}, \varphi, \xi_{i}, \eta^{i}, g\right)$ be an $\mathcal{S}$-manifold. Fixed any integer $p, 1 \leq p \leq s$, we choose $p$ vector fields among the $\xi_{i}$ 's and without loss of generality we can assume that they are $\xi_{1}, \ldots, \xi_{p}$. Then, we consider a new metric $\widetilde{g}$ given by

$$
\widetilde{g}=g-2 \sum_{j=1}^{p} \eta^{j} \otimes \eta^{j} .
$$

For any characteristic vector field $\xi_{i}$, we have $\widetilde{g}\left(\xi_{i}, \xi_{i}\right)=1-2 \sum_{j=1}^{p} \delta_{i}^{j} \delta_{i}^{j}$, so that $\widetilde{g}\left(\xi_{i}, \xi_{i}\right)=-1$ for any $i \leq p$ and $\widetilde{g}\left(\xi_{i}, \xi_{i}\right)=1$ for any $i \geq p+1$, i.e. any $\xi_{i}$ with $i \leq p$ is timelike. Moreover, for any $X, Y \in \operatorname{Im} \varphi, \widetilde{g}(X, Y)=g(X, Y)$ and $\widetilde{g}$ has index $p$.

Proposition 3.1. Let $\left(M^{2 n+s}, \varphi, \xi_{i}, \eta^{i}, g\right)$ be an S-manifold. Fix $p \in\{1, \ldots, s\}$ and the metric $\widetilde{g}$ in (3.1). Then $\left(M^{2 n+s}, \varphi, \xi_{i}, \eta^{i}, \widetilde{g}\right)$ is an indefinite $\mathcal{S}$-manifold of index $p$. 
Proof. Denoting by $\widetilde{\eta}^{i}$ the dual 1-form of $\xi_{i}$ with respect to $\widetilde{g}$, we get

$$
\widetilde{\eta}^{i}(X)=\varepsilon_{i}\left(g\left(X, \xi_{i}\right)-2 \sum_{j=1}^{p} \eta^{j}(X) \delta_{i}^{j}\right) .
$$

Then, $\varepsilon_{i}=1$ for $i \geq p+1$ and $\varepsilon_{i}=-1$ for $i \leq p$ imply $\widetilde{\eta}^{i}=\eta^{i}$ for any $i$. Obviously $\varphi^{2}(X)=-X+\sum_{j=1}^{s} \eta^{j}(X) \xi_{j}$, for any $X \in \mathfrak{X}\left(M^{2 n+s}\right)$. Now we prove the compatibility condition between the metric $\widetilde{g}$ and the $g . f . f$-structure. For any $X, Y \in \mathfrak{X}\left(M^{2 n+s}\right)$, using (3.1), we have

$$
\begin{aligned}
\widetilde{g}(\varphi X, \varphi Y) & =g(\varphi X, \varphi Y)=g(X, Y)-\sum_{i=1}^{s} \eta^{i}(X) \eta^{i}(Y) \\
& =g(X, Y)-2 \sum_{i=1}^{p} \eta^{i}(X) \eta^{i}(Y)+\sum_{i=1}^{p} \eta^{i}(X) \eta^{i}(Y)-\sum_{i=p+1}^{s} \eta^{i}(X) \eta^{i}(Y) \\
& =\widetilde{g}(X, Y)-\sum_{i=1}^{p} \varepsilon_{i} \eta^{i}(X) \eta^{i}(Y)-\sum_{i=p+1}^{s} \eta^{i}(X) \eta^{i}(Y) \\
& =\widetilde{g}(X, Y)-\sum_{i=1}^{s} \varepsilon_{i} \eta^{i}(X) \eta^{i}(Y)
\end{aligned}
$$

The normality condition holds since it does not depend on the metric. Moreover, $\widetilde{\Phi}=\Phi=d \eta^{i}$ for any $i \in\{1, \ldots, s\}$ and $\left(M^{2 n+s}, \varphi, \xi_{i}, \eta^{i}, \widetilde{g}\right)$ turns out to be an indefinite $\mathcal{S}$-manifold of index $p$.

We call such a manifold the associate indefinite $\mathcal{S}$-manifold of index $p$.

Clearly, when $p=1$, we obtain a Lorentz $\mathcal{S}$-manifold.

Now, we look for the link between the Levi-Civita connections determined by $g$ and $\widetilde{g}$ and between the corresponding $\varphi$-sectional curvatures.

Proposition 3.2. Let $\left(M^{2 n+s}, \varphi, \xi_{i}, \eta^{i}, g\right)$ be an $\mathcal{S}$-manifold and $\left(M^{2 n+s}, \varphi, \xi_{i}, \eta^{i}, \widetilde{g}\right)$ the associate indefinite $\mathcal{S}$-manifold of index $p, p \in\{1, \ldots, s\}$. Then the Levi-Civita connections $\nabla$ and $\widetilde{\nabla}$ and the corresponding $\varphi$-sectional curvatures are related by

$$
\begin{gathered}
\widetilde{\nabla}_{X} Y=\nabla_{X} Y+2 \sum_{j=1}^{p}\left\{\eta^{j}(Y) \varphi X+\eta^{j}(X) \varphi Y\right\} \\
\widetilde{K}(X, \varphi X)=K(X, \varphi X)+6 p .
\end{gathered}
$$

Proof. By (3.1), for any $X, Y, Z \in \mathfrak{X}\left(M^{2 n+s}\right)$, we obtain

$$
\widetilde{g}\left(\widetilde{\nabla}_{X} Y, Z\right)=g\left(\widetilde{\nabla}_{X} Y, Z\right)-2 \sum_{j=1}^{p}\left(X\left(\eta^{j}(Y)\right)-d \eta^{j}(X, Y)\right) \eta^{j}(Z),
$$

since

$$
\begin{aligned}
\eta^{j}\left(\widetilde{\nabla}_{X} Y\right) & =\varepsilon_{j} \widetilde{g}\left(\widetilde{\nabla}_{X} Y, \xi_{j}\right)=\varepsilon_{j}\left(X\left(\varepsilon_{j} \eta^{j}(Y)\right)-\widetilde{g}\left(Y, \widetilde{\nabla}_{X} \xi_{j}\right)\right) \\
& =X\left(\eta^{j}(Y)\right)-\varepsilon_{j} \widetilde{g}\left(Y,-\varepsilon_{j} \varphi X\right)=X\left(\eta^{j}(Y)\right)-d \eta^{j}(X, Y) .
\end{aligned}
$$

On the other hand, by the Koszul's formula, with a standard computation, we find

$$
\begin{gathered}
\widetilde{g}\left(\widetilde{\nabla}_{X} Y, Z\right)=g\left(\nabla_{X} Y, Z\right)-\sum_{j=1}^{p}\left\{\eta^{j}(Z)\left(-2 d \eta^{j}(X, Y)+2 X\left(\eta^{j}(Y)\right)\right)\right. \\
\left.-2 \eta^{j}(Y) d \eta^{j}(Z, X)-2 \eta^{j}(X) d \eta^{j}(Z, Y)\right\} .
\end{gathered}
$$

Comparing (3.4) and (3.5), using also $\Phi=d \eta^{j}$, we get

$$
g\left(\widetilde{\nabla}_{X} Y, Z\right)=g\left(\nabla_{X} Y, Z\right)+2 \sum_{j=1}^{p}\left\{\eta^{j}(Y) g(Z, \varphi X)+\eta^{j}(X) g(Z, \varphi Y)\right\},
$$


which implies (3.2).

Now, we have $\widetilde{\nabla}_{X} Y=\nabla_{X} Y$, for any $X, Y \in \operatorname{Im} \varphi$. We consider $X \in \operatorname{Im} \varphi$ such that $\|X\|_{g}=\|X\|_{\tilde{g}}=1$ and since $\eta^{j}\left(\nabla_{\varphi X} \varphi X\right)=-g\left(\varphi X, \nabla_{\varphi X} \xi_{j}\right)=g\left(\varphi X, \varphi^{2} X\right)=0$ and $\eta^{j}\left(\nabla_{X} \varphi X\right)=-g\left(\varphi X, \nabla_{X} \xi_{j}\right)=g(\varphi X, \varphi X)=1$, we get

$$
\begin{gathered}
\widetilde{\nabla}_{X} \widetilde{\nabla}_{\varphi X} \varphi X=\widetilde{\nabla}_{X} \nabla_{\varphi X} \varphi X=\nabla_{X} \nabla_{\varphi X} \varphi X \\
\widetilde{\nabla}_{\varphi X} \widetilde{\nabla}_{X} \varphi X=\widetilde{\nabla}_{\varphi X} \nabla_{X} \varphi X=\nabla_{\varphi X} \nabla_{X} \varphi X-2 p X .
\end{gathered}
$$

Furthermore,

$$
\begin{aligned}
\widetilde{\nabla}_{[X, \varphi X]} \varphi X & =\nabla_{[X, \varphi X]} \varphi X+2 \sum_{j=1}^{p} \eta^{j}([X, \varphi X]) \varphi^{2} X \\
& =\nabla_{[X, \varphi X]} \varphi X+4 \sum_{j=1}^{p} d \eta^{j}(X, \varphi X) X=\nabla_{[X, \varphi X]} \varphi X-4 p X
\end{aligned}
$$

From (3.6), (3.7) and (3.8) we get

$$
\widetilde{R}_{X \varphi X} \varphi X=R_{X \varphi X} \varphi X+6 p X
$$

and (3.3) follows.

Remark 3.1. The inverse transformation of (3.1), $g=\widetilde{g}+2 \sum_{j=1}^{p} \eta^{j} \otimes \eta^{j}$, allows to construct $\mathcal{S}$-manifolds starting from indefinite $\mathcal{S}$-manifolds, whose index only depends on the casual character of $p$ characteristic vector fields among the $\xi_{i}$ 's. Furthermore, if we start from an indefinite $\mathcal{S}$-manifold of index $2 q, q \leq n$, with all the $\xi_{i}$ 's spacelike, then, using the same transformation (3.1) of the metric, we get an associate indefinite $\mathcal{S}$-manifold of index $2 q+p$ and (3.2) and (3.3) hold as well. Namely, the proof goes on as in Proposition 3.2 with the remark that considering $\widetilde{g}(X, X)=g(X, X)=\varepsilon_{X}= \pm 1$ for a non null vector, last terms in (3.7), (3.8) and (3.9) become $-2 p \varepsilon_{X} X,-4 p \varepsilon_{X} X$ and $6 p \varepsilon_{X} X$, respectively.

Again, looking at the relation between the $\varphi$-sectional curvatures, it is clear that the behaviors of an $\mathcal{S}$ manifold and its associated Lorentz $\mathcal{S}$-manifold are strictly related as the following theorem shows.

Theorem 3.3. Let $\left(M^{2 n+s}, \varphi, \xi_{i}, \eta^{i}, g\right)$ be an $\mathcal{S}$-manifold, $\left(M^{2 n+s}, \varphi, \xi_{i}, \eta^{i}, \tilde{g}\right)$ the associated Lorentz $\mathcal{S}$-manifold. Then the $\mathcal{S}$-manifold has constant $\varphi$-sectional curvature $c \in \mathbb{R}$ if and only if the Lorentz $\mathcal{S}$-manifold has constant $\varphi$-sectional curvature $\tilde{c}=c+6$ and we have $c>-3 s \Leftrightarrow \tilde{c}>-3 s+6$.

Now, considering a compact $\mathcal{S}$-manifold $M^{2 n+s}$ and the associated indefinite $\mathcal{S}$-manifold with signature $(2 n+s-p, p)$, with $2 p \leq 2 n+s$, then $\left(M^{2 n+s}, \tilde{g}\right)$ is geodetically complete, by Theorem 3.2. In particular this holds for $p \leq \frac{s}{2}$.

\section{D-homothetic transformations}

In the context of contact metric manifolds $\left(M^{2 n+1}, \varphi, \xi, \eta, g\right)$ a $D_{a}$-homothetic deformation, $a>0$, is a change of the structure tensors $(\varphi, \xi, \eta, g)$ as follows

$$
\varphi^{\prime}:=\varphi, \quad \xi^{\prime}:=\frac{1}{a} \xi, \quad \eta^{\prime}:=a \eta, \quad g^{\prime}:=a g+a(a-1) \eta \otimes \eta .
$$

This notion was introduced by Tanno, [18], in the contact metric case and the class of Sasakian structures is preserved by such deformations.

One can easily extend such a notion to more general contexts.

On a metric ( $g . f . f)$-manifold $\left(M^{2 n+s}, \varphi, \xi_{i}, \eta^{i}, g\right)$, by a $D_{a}$-homothetic deformation of constant $a$ we mean the following change of the structure tensors, [8]:

$$
\tilde{\varphi}=\varphi, \tilde{\xi}_{i}=\frac{1}{a} \xi_{i}, \tilde{\eta}^{i}=a \eta^{i}, \tilde{g}=a g+a(a-1) \sum_{j=1}^{s} \eta^{j} \otimes \eta^{j}
$$

www.iejgeo.com 
and again the class of $\mathcal{S}$-manifolds is preserved by such deformations.

Moreover, [7], the Levi-Civita connections of $g$ and $\tilde{g}$ on an $\mathcal{S}$-manifold are linked by

$$
\tilde{\nabla}_{X} Y=\nabla_{X} Y+(1-a)(\bar{\eta}(Y) \varphi X+\bar{\eta}(X) \varphi Y)
$$

where $\bar{\eta}=\sum_{i=1}^{s} \eta^{i}$.

For the $\varphi$-sectional curvatures, from

$$
\tilde{R}_{X \varphi X} \varphi X=R_{X \varphi X} \varphi X-3 s(a-1) X
$$

one obtains

$$
\tilde{K}(X, \varphi X)=\frac{1}{a}(K(X, \varphi X)-3 s(a-1)) .
$$

Then, if $\left(M^{2 n+s}, \varphi, \xi_{i}, \eta^{i}, g\right)$ is an $S$-manifold with constant $\varphi$-sectional curvature $c$, we obtain that the $D_{a}$ - homothetic $\mathcal{S}$-manifold $\left(M^{2 n+s}, \varphi, \tilde{\xi}_{i}, \tilde{\eta}^{i}, \tilde{g}\right)$ has constant $\varphi$-sectional curvature $\tilde{c}$ given by

$$
\tilde{c}=\frac{1}{a}(c-3 s(a-1)) .
$$

Note that the three subclasses defined by $c>-3 s, c=-3 s$ and $c<-3 s$ are invariant by $D_{a}$-homothetic deformations, $a>0$. Therefore, any $\mathcal{S}$-manifold of $\varphi$-sectional curvature $c>-3 s$, choosing $a=\frac{c+3 s}{1+3 s}>0$ is $D$-homothetic to an $\mathcal{S}$-manifold of $\varphi$-sectional curvature 1 . By the contrary, a $D_{a}$-homothetic deformation with $a<0$ maps the subclass defined by $c>-3 s$ in that defined by $c<-3 s$ and vice versa.

Now, we consider indefinite $\mathcal{S}$-manifolds.

Let $\left(\varphi, \xi_{i}, \eta^{i}, g\right), \quad 1 \leq i \leq s$, be an indefinite g.f.f-structure and $\alpha \in \mathbb{R}^{*}$. We mean by a $D$-homothetic transformation of constant $\alpha$ a change of the structure tensors as follows:

$$
\tilde{\varphi}=\varphi, \quad \tilde{\xi}_{i}=\frac{1}{\alpha} \xi_{i}, \quad \tilde{\eta}^{i}=\alpha \eta^{i}, \quad \tilde{g}=\alpha g+\alpha(\alpha-1) \sum_{i=1}^{s} \varepsilon_{i} \eta^{i} \otimes \eta^{i} .
$$

Remark 4.1. One can directly verify that a structure $\left(\tilde{\varphi}, \tilde{\xi}_{i}, \tilde{\eta}^{i}, \tilde{g}\right)$ obtained by a $D$-homothetic transformation from an indefinite g.f.f-structure $\left(\varphi, \xi_{i}, \eta^{i}, g\right)$ maintains the same properties of $\left(\varphi, \xi_{i}, \eta^{i}, g\right)$. Moreover, the transformation preserves the signature of the metrics $g$ and $\tilde{g}$ on $\operatorname{ker} \varphi$, when $\alpha>0$ and the signature of the metrics on $\operatorname{Im} \varphi$ is again preserved. By the contrary, if $\alpha<0$, the transformation changes the spacelike vector fields into timelike ones and vice versa so that the signatures change.

The link between the Levi-Civita connections $\nabla$ and $\tilde{\nabla}$ of $g$ and $\tilde{g}$ is given by:

$$
\tilde{\nabla}_{X} Y=\nabla_{X} Y+(1-\alpha)(\bar{\eta}(X) \varphi Y+\bar{\eta}(Y) \varphi X)
$$

where $\bar{\eta}=\sum_{i=1}^{s} \varepsilon_{i} \eta^{i}$. Consider $X \in \operatorname{Im} \varphi$ such that $g(X, X)=\varepsilon_{X}$, by the above formula it follows that $\tilde{R}_{X \varphi X} \varphi X=R_{X \varphi X} \varphi X-3 \varepsilon \varepsilon_{X}(\alpha-1) X$, where $\varepsilon=\sum_{i=1}^{s} \varepsilon_{i}$ and this yields

$$
\tilde{K}(X, \varphi X)=\frac{1}{\alpha}\left(K(X, \varphi X)-3 \varepsilon \varepsilon_{X}(\alpha-1)\right) .
$$

\section{Local semi-Riemannian decomposition of indefinite $\mathcal{S}$-manifolds}

Firstly we recall that an almost contact metric structure $(\varphi, \xi, \eta, g)$ on a manifold $M^{2 n+1}$ is called an $\alpha$-Sasakian structure, $\alpha>0$, if it is normal and its Sasaki 2-form $\Phi$ verifies $d \eta=\alpha \Phi$. In [8] the authors have proved that an $\mathcal{S}$-manifold $\left(M^{2 n+s}, \varphi, \xi_{i}, \eta^{i}, g\right)$ is locally a Riemannian product of a $\sqrt{s}$-Sasakian manifold and an $(s-1)$-dimensional flat manifold.

We are going to discuss a similar problem in Lorentz context, remarking that the distributions considered by the authors in [8] have to be adapted to the Lorentz case because they are neither orthogonal nor parallel with respect to the Levi-Civita connection when the metric is Lorentz. Following [1] we recall some fundamental notion about distributions and foliations in semi-Riemannian manifolds. A distribution $D$ on $M$ is said to be parallel with respect to a linear connection $\nabla$ if $D$ is invariant under parallel transport $\tau$, i.e. $\tau_{\sigma}\left(D_{x}\right)=D_{y}$ for 
all $x, y \in M$ and all piecewise smooth paths $\sigma$ from $x$ to $y$. In [1, pg 155] it is stated that a distribution $D$ on a manifold $M$ is parallel with respect to a linear connection $\nabla$ on $M$ if and only if $\nabla$ is a connection adapted to $D$, that is $\nabla_{X} Y \in \Gamma(D)$ for any $X \in \Gamma(T M)$ and $Y \in \Gamma(D)$. Moreover, a foliation on a semi-Riemannian manifold is said to be non-degenerate (resp.: parallel) if its tangent distribution is non-degenerate (resp.: parallel). It is well known that a semi-Riemannian manifold admitting a parallel non-degenerate foliation admits a local semi-Riemannian product decomposition, [1, Theorem 4.2, pg 165],[21], and now we apply this result to a Lorentz $\mathcal{S}$-manifold.

Let $\left(M^{2 n+s}, \varphi, \xi_{i}, \eta^{i}, g\right)$ be a Lorentz $\mathcal{S}$-manifold. By the metric compatibility condition it is clear that the metric index can only depend on the causal character of the characteristic vector fields, thus one of them has to be timelike and, to fix the ideas, let us assume that $\xi_{1}$ is timelike.

Theorem 5.1. Let $\left(M^{2 n+s}, \varphi, \xi_{i}, \eta^{i}, g\right)$ be a Lorentz $\mathcal{S}$-manifold with $\xi_{1}$ timelike. Then $\left(M^{2 n+s}, g\right)$ is locally a semiRiemannian product of a $\sqrt{s-2}$-Sasakian manifold and of an $(s-1)$-dimensional flat manifold.

Proof. Let us consider $\bar{\xi}=\sum_{i=1}^{s} \xi_{i}$ and $\bar{\eta}=\sum_{j=1}^{s} \varepsilon_{j} \eta^{j}$, putting $\tilde{\xi}_{i}=\varepsilon_{1} \xi_{1}-\xi_{i}, i \in\{2, \ldots, s\}$, we get a basis of $\operatorname{span}\{\bar{\xi}\}^{\perp_{\text {ker }}}$. Note that any $\tilde{\xi}_{i}$ is lightlike. Hence $T M^{2 n+s}=(\operatorname{Im} \varphi \oplus \operatorname{span}\{\bar{\xi}\}) \oplus \operatorname{span}\left\{\tilde{\xi}_{2}, \ldots, \tilde{\xi}_{s}\right\}$ and by the properties of indefinite $\mathcal{S}$-manifolds it is easy to show that the distributions $D_{1}=(\operatorname{Im} \varphi \oplus \operatorname{span}\{\bar{\xi}\})$ and $D_{2}=\operatorname{span}\left\{\tilde{\xi}_{2}, \ldots, \tilde{\xi}_{s}\right\}$ are integrable, parallel with respect to the Levi-Civita connection and they are not degenerate. In fact the distribution $D_{1}$ is not degenerate because $\operatorname{Im} \varphi$ and $\operatorname{span}\{\bar{\xi}\}$ are orthogonal and $g(\bar{\xi}, \bar{\xi})=s-2 \neq 0$, then $D_{2}=D_{1}^{\perp}$ is not degenerate. Therefore, [1], $\left(M^{2 n+s}, g\right)$ is locally a semi-Riemannian product of integral submanifolds $M_{1}$ and $M_{2}$ of $D_{1}$ and $D_{2}$, respectively. Let $\tilde{\xi}=\frac{1}{\sqrt{s-2}} \bar{\xi}$ and $\tilde{\eta}=\frac{1}{\sqrt{s-2}} \bar{\eta}$. It is easy to verify that $\left(M_{1}, \varphi, \tilde{\xi}, \tilde{\eta}, g\right)$ is a normal contact metric manifold. Finally, a computation shows that $d \tilde{\eta}=\frac{1}{\sqrt{s-2}} d \bar{\eta}=\frac{1}{\sqrt{s-2}} \sum_{i=1}^{s} \varepsilon_{i} d \eta^{i}=\sqrt{s-2} \Phi$. So $\left(M_{1}, \varphi, \tilde{\xi}, \tilde{\eta}, g\right)$ is a $\sqrt{s-2}$-Sasakian manifold. Obviously $M_{2}$ is an $(s-1)$-dimensional flat manifold.

Let $\left(M^{2 n+s}, \varphi, \xi_{i}, \eta^{i}, g\right)$ be an indefinite $\mathcal{S}$-manifold and $p$ and $q$ two integers. We suppose that the metric index $\nu(g)=2 q+p$ depends even on the characteristic vector fields and, to fix the ideas, let us assume that $\xi_{1}, \ldots, \xi_{p}$ are timelike.

Theorem 5.2. Let $\left(M^{2 n+s}, \varphi, \xi_{i}, \eta^{i}, g\right)$, be an indefinite $\mathcal{S}$-manifold with $g$ of index $2 q+p, p \neq \frac{s}{2}$ and $\xi_{1}, \ldots, \xi_{p}$ timelike vector fields. Then $\left(M^{2 n+s}, g\right)$ is locally a semi-Riemannian product of an indefinite $\sqrt{|s-2 p|}$-Sasakian manifold and an $(s-1)$-dimensional flat manifold.

Proof. Let $\bar{\xi}=\sum_{i=1}^{s} \xi_{i}, \bar{\eta}=\sum_{j=1}^{s} \varepsilon_{j} \eta^{j}$. Then putting $\tilde{\xi}_{i}=\varepsilon_{1} \xi_{1}-\varepsilon_{i} \xi_{i}, i \in\{2, \ldots, s\}$, we get a basis of $\operatorname{span}\{\bar{\xi}\}^{\perp_{\text {ker }}}$ and $T M^{2 n+s}=(\operatorname{Im} \varphi \oplus \operatorname{span}\{\bar{\xi}\}) \oplus \operatorname{span}\left\{\tilde{\xi}_{2}, \ldots, \tilde{\xi}_{s}\right\}$. The integrable two distributions $D_{1}=\operatorname{Im} \varphi \oplus \operatorname{span}\{\bar{\xi}\}$ and $D_{2}=\operatorname{span}\left\{\tilde{\xi}_{2}, \ldots, \tilde{\xi}_{s}\right\}$ are parallel with respect to the Levi-Civita connection. Moreover, the distribution $D_{1}$ is not degenerate when $p \neq \frac{s}{2}$ because $g(\bar{\xi}, \bar{\xi})=s-2 p$, and $D_{2}$ is orthogonal to $D_{1}$. Therefore, using the result of [1], $\left(M^{2 n+s}, g\right)$ is locally a semi-Riemannian product of integral submanifolds $M_{1}$ and $M_{2}$ of $D_{1}$ and $D_{2}$ respectively. Let $\tilde{\xi}=\frac{1}{\sqrt{|s-2 p|}} \bar{\xi}$ and $\tilde{\eta}=\frac{\varepsilon_{p}}{\sqrt{|s-2 p|}} \bar{\eta}$, where $\varepsilon_{p}=1$ if $2 p<s$ and $\varepsilon_{p}=-1$ otherwise. It is easy to verify that $\left(M_{1}, \varphi, \tilde{\xi}, \tilde{\eta}, g\right)$ is a normal contact metric manifold. Finally, with a straightforward computation we obtain that $d \tilde{\eta}=\frac{\varepsilon_{p}}{\sqrt{|s-2 p|}} d \bar{\eta}=\frac{\varepsilon_{p}}{\sqrt{|s-2 p|}} \sum_{i}^{s} \varepsilon_{i} d \eta^{i}=\sqrt{|s-2 p|} \Phi$. Therefore, $\left(M_{1}, \varphi, \tilde{\xi}, \tilde{\eta}, g\right)$ is an indefinite $\sqrt{|s-2 p|}$-Sasakian manifold. On the other hand $M_{2}$ is obviously an $(s-1)$-dimensional flat manifold.

We remark that the above theorems do not work in the case $s=2$, or more generally, $s$ even and $p=\frac{s}{2}$. We shall discuss them in next section.

\section{The case of special indefinite $\mathcal{S}$-manifolds}

We recall that an indefinite $\mathcal{S}$-manifold is said to be special if $\varepsilon=0$, i.e. $s=2 p$ and, without loss of generality, we suppose that $\xi_{1}, \ldots, \xi_{p}$ are timelike vector fields, while $\xi_{p+1}, \ldots, \xi_{2 p}$ are spacelike vector fields, [4]. A known example of special $\mathcal{S}$-manifold is given in [5] for the group $U(2)$. The 4-dimensional manifold $U(2)$ has a structure $\left(\varphi, \xi_{1}, \xi_{2}, \eta^{1}, \eta^{2}, g\right)$ of $\mathcal{S}$-manifold described in [9] and the $\varphi$-sectional curvatures are

$$
K(X, \varphi X)=-2, K\left(X, \xi_{1}\right)=K\left(Y, \xi_{1}\right)=1, K\left(X, \xi_{2}\right)=K\left(Y, \xi_{2}\right)=1 .
$$


We can define a left-invariant Lorentz metric $\tilde{g}$, with $\xi_{1}$ timelike, obtaining a Lorentzian $\mathcal{S}$-structure of constant $\varphi$-sectional curvature $\tilde{K}(X, \varphi X)=-2+6=4$. Finally, $\tilde{K}\left(X, \xi_{1}\right)=\tilde{K}\left(Y, \xi_{1}\right)=-1$ and $\tilde{K}\left(X, \xi_{2}\right)=\tilde{K}\left(Y, \xi_{2}\right)=1$. Note that $\xi_{1}+\xi_{2}$ is a lightlike vector field and, as proved in [5], the Lorentz $\mathcal{S}$-manifold $\left(U(2), \varphi, \xi_{1}, \xi_{2}, \eta^{1}, \eta^{2}, \tilde{g}\right)$ is foliated by Reinhart lightlike manifolds.

Now we recall that a submanifold $M$ of a semi-Riemannian manifold $(\bar{M}, \bar{g})$ is said to be lightlike if the induced metric $g$ on $M$ is degenerate [10]. Moreover, let $(M, g)$ be a lightlike manifold of dimension $m$. Suppose that $\operatorname{Rad}(T M)$ is an integrable distribution of rank $r>0$. Then $M$ is called a Reinhart lightlike manifold if locally there exists a system of coordinates of $M,\left(U, x^{1}, \ldots, x^{m}\right)$, such that $\frac{\partial g_{i j}}{\partial x^{\alpha}}=0$ for $i, j \in\{r+1, \ldots, m\}$ and $\alpha \in\{1, \ldots, r\}$, where $x^{\alpha}$ are the coordinates of a leaf $L$ of $\operatorname{Rad}(T M)$ [10].

More in general, we state the following result.

Theorem 6.1. Any Lorentz $\mathcal{S}$-manifold $\left(M^{2 n+2}, \varphi, \xi_{i}, \eta^{i}, g\right), 1 \leq i \leq 2$, is foliated by Reinhart lightlike manifolds.

Proof. We assume $\xi_{1}$ timelike. We put $\bar{\xi}=\xi_{1}+\xi_{2}$ and $\bar{\eta}=-\eta^{1}+\eta^{2}$. Putting $\bar{\xi}_{2}=-\xi_{1}+\xi_{2}$, we get $g\left(\bar{\xi}, \bar{\xi}_{2}\right)=2$. Note that $\bar{\xi}$ and $\bar{\xi}_{2}$ are lightlike. Hence $T M^{2 n+2}$ decomposes as $T M^{2 n+2}=(\operatorname{Im} \varphi \oplus \operatorname{span}\{\bar{\xi}\}) \oplus \operatorname{span}\left\{\bar{\xi}_{2}\right\}$ and by the properties of indefinite $\mathcal{S}$-manifolds, in particular using (3.2), with $p=1$, it is easy to show that the distributions $D_{1}=(\operatorname{Im} \varphi \oplus \operatorname{span}\{\bar{\xi}\})$ and $D_{2}=\operatorname{span}\left\{\bar{\xi}_{2}\right\}$ are integrable, and degenerate. Therefore, $M^{2 n+2}$ is locally a product of integral submanifolds $M_{1}$ and $M_{2}$ of $D_{1}$ and $D_{2}$, respectively. It is foliated by the integral submanifolds of $D_{1}$, which are lightlike hypersurfaces. Then, for such an $M_{1}$ one considers the radical distribution $\operatorname{Rad}\left(T M_{1}\right)$, such that for any $p \in M_{1}$

$$
\operatorname{Rad} T_{p} M_{1}=\left\{V \in T_{p} M_{1} \mid g_{p}(V, W)=0 \text { for all } W \in T_{p} M_{1}\right\}=T_{p} M_{1}^{\perp} \cap T_{p} M_{1} .
$$

Any decomposition $T_{p} M_{1}=\operatorname{Rad} T_{p} M_{1} \perp S\left(T_{p} M_{1}\right)$ gives rise to a non-degenerate distribution $S\left(T M_{1}\right)$ on $M_{1}$, called a screen distribution. We recall the following theorem [10, p.79].

Theorem 6.2. Let $(M, g, S(T M))$ be a lightlike hypersurface of a semi-Riemannian manifold $(\bar{M}, \bar{g})$. Then there exists a unique rank one vector subbundle $\operatorname{lt}(M)$ of $T \bar{M}$, with base space $M$, such that for any non-zero section $E$ of $T M^{\perp}$ on a coordinate neighborhood $\mathcal{U} \subset M$, there exists a unique section $N$ of $\operatorname{ltr}(M)$ on $\mathcal{U}$ satisfying: $\bar{g}(N, E)=1, \bar{g}(N, N)=0, \bar{g}(N, W)=0$ for any $W \in \Gamma(S(T M) \mid \mathcal{u})$. The vector bundle ltr $(M)$ is called the lightlike transversal vector bundle of $M$ with respect to $S(T M)$.

Now, we have $\operatorname{Rad}\left(T M_{1}\right)=\operatorname{span}\left\{\xi_{1}+\xi_{2}\right\}$ and $S\left(T M_{1}\right)=\operatorname{Im} \varphi$. We construct a global section $N$ of $l \operatorname{tr}\left(M_{1}\right)$. Being $S\left(T M_{1}\right)^{\perp}=\operatorname{span}\left\{\xi_{1}, \xi_{2}\right\}$, we choose $E=\xi_{1}+\xi_{2}$ so that the vector field $N=\frac{1}{2}\left(\xi_{2}-\xi_{1}\right)$ verifies the conditions in Theorem 6.2. Moreover, it is easy to verify that $\operatorname{Rad}\left(T M_{1}\right)$ is a Killing distribution so that, via Theorem 5.1, p.49 in [10], $M_{1}$ is a Reinhart lightlike manifold.

We discuss the same situation when $s=2 p$.

Theorem 6.3. Any special indefinite $\mathcal{S}$-manifold $\left(M^{2 n+s}, \varphi, \xi_{i}, \eta^{i}, g\right), i \in\{1, s\}, s=2 p$ is foliated by Reinhart lightlike manifolds.

Proof. Without loss of generality, we assume $\xi_{1}, \ldots, \xi_{p}$ timelike. We put $\bar{\xi}=\sum_{i=1}^{s} \xi_{i}, \bar{\eta}=\sum_{j=1}^{s} \varepsilon_{j} \eta^{j}$. Then putting $\tilde{\xi}_{i}=\varepsilon_{1} \xi_{1}-\varepsilon_{i} \xi_{i}$, for all $i \in\{2, \ldots, s\}$, we get a basis of $\operatorname{span}\{\bar{\xi}\}^{\perp_{\text {ker }}}$ and $T M^{2 n+2 p}$ decomposes as $T M^{2 n+2 p}=(\operatorname{Im} \varphi \oplus \operatorname{span}\{\bar{\xi}\}) \oplus \operatorname{span}\left\{\tilde{\xi}_{2}, \ldots, \tilde{\xi}_{s}\right\}$.

Note that $\bar{\xi}$ is lightlike and by the properties of indefinite $\mathcal{S}$-manifolds, in particular using (3.2), it is easy to show that the distributions $D_{1}=(\operatorname{Im} \varphi \oplus \operatorname{span}\{\bar{\xi}\})$ and $D_{2}=\operatorname{span}\left\{\tilde{\xi}_{2}, \ldots, \tilde{\xi}_{s}\right\}$ are integrable, and degenerate. Therefore, $M^{2 n+2 p}$ is locally a product of integral submanifolds $M_{1}$, of dimension $2 n+1$, and $M_{2}$ of dimension $2 p-1$, of $D_{1}$ and $D_{2}$, respectively. Moreover $M^{2 n+2 p}$ is foliated by the integral submanifolds of $D_{1}$, which are lightlike. Then, for an integral submanifolds $M_{1}$ one considers the radical distribution $\operatorname{Rad}\left(T M_{1}\right)$, such that for any $p \in M_{1}$

$$
\operatorname{Rad} T_{p} M_{1}=\left\{V \in T_{p} M_{1} \mid g_{p}(V, W)=0 \text { for all } W \in T_{p} M_{1}\right\}=T_{p} M_{1}^{\perp} \cap T_{p} M_{1} .
$$

Any decomposition $T_{p} M_{1}=\operatorname{Rad} T_{p} M_{1} \perp S\left(T_{p} M_{1}\right)$ gives rise to a non-degenerate distribution $S\left(T M_{1}\right)$ on $M_{1}$, called a screen distribution.

Now, we have $\operatorname{Rad}\left(T M_{1}\right)=\operatorname{span}\{\bar{\xi}\}$ and $S\left(T M_{1}\right)=\operatorname{Im} \varphi$. We construct a global section $N$ of $\operatorname{ltr}\left(M_{1}\right)$. Being $S\left(T M_{1}\right)^{\perp}=\operatorname{span}\left\{\xi_{1}, \ldots, \xi_{s}\right\}$, we choose $E=\xi_{1}+\xi_{2}$ so that the vector field $N=\frac{1}{2}\left(\xi_{2}-\xi_{1}\right)$ verifies the conditions in Theorem 6.2. Moreover, via Theorem 5.1, p.49 in [10], since $\operatorname{Rad}\left(T M_{1}\right)$ is a Killing distribution, we get that $M_{1}$ is a Reinhart lightlike manifold. 


\section{References}

[1] Bejancu, Aurel and Farran, Hani Reda, Foliations and Geometric Structures, Springer Verlag, Berlin, 2006.

[2] Blair, D.E., Geometry of manifolds with structural group $\mathcal{U}(n) \times \mathcal{O}(s)$, J. Differential Geometry 4 (1970), 155-167.

[3] Blair, D.E., Riemannian geometry of contact and symplectic manifolds, Progr. Math., 203, Birkhäuser Boston, Boston, MA, 2002.

[4] Brunetti, L. and Pastore, A.M., Curvature of a class of indefinite globally framed f-manifolds, Bull. Math. Soc. Sci. Math. Roumanie 5199 (2008), no.3, 183-204.

[5] Brunetti, L. and Pastore, A.M., Examples of indefinite globally framed $f$-structures on compact Lie groups, Publ. Math. Debrecen 80 1-2 (2012), 215-234.

[6] Brunetti, L. and Pastore, A.M., On the classification of Lorentzian Sasaki space forms, Publications de l'Institut Mathématique Nouvelle série 94 (2013), no.108, 163-168.

[7] Cappelletti Montano, B. and Di Terlizzi L., D-homothetic transformations for a generalization of contact metric manifolds, Bull. Belg. Math. Soc. 14 (2007), 277-289.

[8] Di Terlizzi, L. and Pastore, A.M., K-manifolds locally described by Sasaki manifolds, An. St. Univ. Ovidius Constanta 21 (2013), no.3, 269-287.

[9] Di Terlizzi, L. and Konderak, J.J., Examples of a generalization of contact metric structures on fibre bundles, J. of Geometry 87 (2007), $31-49$.

[10] Duggal, Krishan L. and Bejancu, Aurel, Lightlike submanifolds of semi-Riemannian manifolds and applications. Kluwer Acad. Publ., Dordrecht, 1996.

[11] Goldberg, S.I. and Yano, K., On normal globally framed f-manifolds, Tôhoku Math. J., 22 (1970), 362-370.

[12] Goldberg, S.I., On the existence of manifolds with an $f$-structure, Tensor, N. S. 26 (1972), 323-329.

[13] Guediri, M. and Lafontaine, J., Sur la complétude des varietés pseudoriemanniennes, J. Geom. Phys. 15 (1995), 150-158.

[14] Kobayashi,S. and Nomizu,K., Foundations of Differential Geometry, Vol. I, II Interscience Publish., New York, $1963,1969$.

[15] Kobayashi, M., and Tsuhiya, S., Invariant submanifolds of an $f$-manifold with complemented frames, Kodai Math. Semin. Rep. 24 (1972), $430-450$.

[16] O’Neill, B., Semi-Riemannian geometry. Academic Press, New York, 1983.

[17] Takahashi, T., Sasakian manifold with pseudo-Riemannian metric, Tôhoku Math. J. 21 (1969), no.2, 271-290.

[18] Tanno, S., The topology of contact Riemannian manifolds, Illinois J. Math. 12 (1968), 700-717.

[19] Tanno, S., Sasakian manifolds with constant $\phi$-holomorphic sectional curvature, Tôhoku Math. J. 21 (1969), no.3, 501-507.

[20] Yano, K., On a structure defined by a tensor field of type (1,1) satisfying $f^{3}+f=0$, Tensor (N.S.) 14 (1963), 99-109.

[21] Wu, H., On the de Rham decomposition theorem, Illinois J. Math. 8 (1964), 291-311.

\section{Affiliations}

Letizia BRUnetTI, ANNAMARIA PASTORE

ADDRESS: University of Bari Aldo Moro, Department of Mathematics, 70125, Bari-Italy.

E-MAIL: letybmail@hotmail.it, annamaria.pastore@uniba.it 